\title{
Endo Bronchial Hamartoma Causing Obstructive Changes
}

\author{
Dr. M. Vijayasree M D ${ }^{1}$, Dr.I .V.Renuka M. D², Dr. G. Saila bala M.D ${ }^{3}$, \\ Dr. K. Nageswara rao M.D ${ }^{4}$ \\ ${ }^{1}$ Assoc. Professor, Dept. of Pathology, Guntur Medical College, Guntur, India \\ ${ }^{2}$ Professor, Dept. of pathology, Guntur Medical College, Guntur, India \\ ${ }^{3}$ Professor, Dept. of pathology, Guntur Medical College, Guntur, India \\ ${ }^{4}$ Assistant Professor, Dept. of pathology, Guntur Medical College, Guntur, India
}

\begin{abstract}
Pulmonary hamartomas are usually an incidental findings and range in size from $1 \mathrm{~cm}$ to $8 \mathrm{~cm}$ in diameter. We report a case of an endobronchial hamartoma in a 43 year old female presented with progressive breathlessness since one year,pleuritic chest pain,cough,reeling sensation and decreased hearing in left ear since 3 months.patient was a known case of pulmonary tuberculosis and past history of left side pleural effusion present
\end{abstract}

\section{Introduction}

Hamartomas also known as mesenchymomas,benign neoplasm composed of cartilage,fat,fibromyxoid tissue,smooth muscle and bone.Hamartomas can be parenchymal (or) endobronchial in location.The parenchymal lesions are asymptomatic. The endobronchial tumors usually present with new onset of respiratory symptomas.Here we report a case of endobronchial hamartoma.

\section{CASE REPORT}

A previously healthy 43 year old female was investigated for shortness of breath. on chest examination the left side was dull on percussion with diminished breath sounds. Chest radiography showing opacity in the left lower and middle zones.

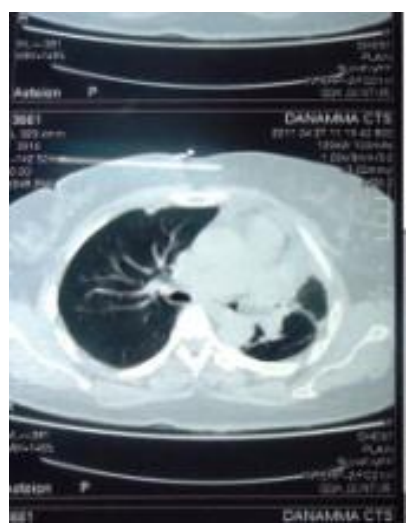

Figure $1 \mathrm{CT}$ demonstrates the soft tissue mass noted in the left main bronchus

CT scan revealed old fibrotic strands in the left lung,no active lesion,soft tissue density noted in the left main bronchus extending in to lingular and lower bronchus with severe obstruction.Flexible fibrooptic bronchoscopy showed a well defind smooth mucosa lined polypoid growth obstructing left main bronchus at its bifurcation.

A clinical diagnosis of bronchial carcinoid (or) adenoma was made.All the hematological and biochemical parameters were with in normal limits.We received a pneumonectomy specimen of size $16 \times 12 \times 4 \mathrm{~cm}$,external surface dark brown and bronchus filled with a yellowish mass of size $3 \times 2 \times 1 \mathrm{~cm}$. On cut section mass is yellowish and thinly capsculated.The post operative recovery was uneventful and the patient was discharged on the ninth post operative day.The final histology of tumor was a endobronchial hamartoma composed of mostly adipose tissue, mucus glands, islands of cartilage, bone and bone marrow. 


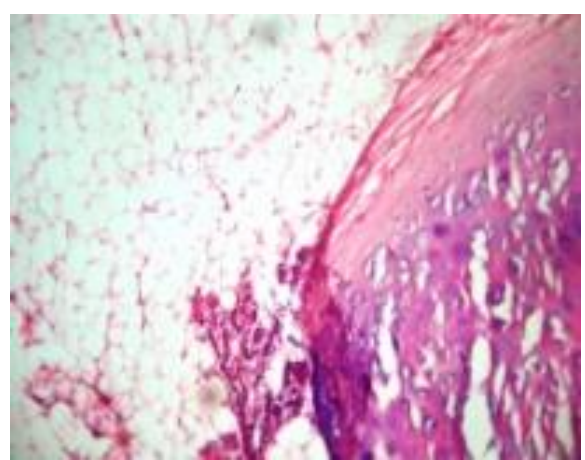

Figure 2. H\&E X400;Components shown in detail are adipose tissue and cartilage

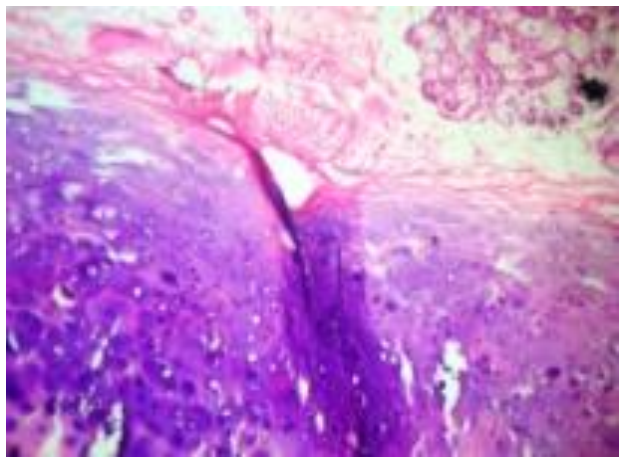

Figure 3 H\&E X400;A lobular mass of mature hyaline cartilage

\section{Discussion:}

pulmonary hamartomas (or) mesenchymomas are the most common type of benign lung tumors (1) Most of them are parenchymal lesions. The endobronchial lesions making upto only about $10 \%$.The peak incidence is between fifth and seventh decade with a male preponderance ${ }^{(2)}$. The parenchymal lesions are usually an incidental radiological findings of a rounded homogenous opacity in the periphery, where as endo bronchial lesions had pulmonary symptoms like cough,haemoptysis,dysponea on exertion and feverendobronchial hamartomas are usually pedunculated and originate from the proximal portion of the bronchial tree.65\% of endobronchial hamartomas occur on the right side and 35\% on left side.In a series of 154 cases of pulmonary hamartoma studied by Van den Bosch et al only 12 were endobronchial while the rest were peripheral(parenchymal) lesions. They found a male preponderance with the peak incidence in sixth decade. ${ }^{(3)}$ IN 43 out of 49 patients reported by Ramirez - Chavez et al The hamartomas were peripheral while only 6 were endobronchial with obstructive changes ${ }^{(4)}$ Tomashefski compared 17 benign endobronchial tumors with 147 intra parenchymal hamartomas and concluded that the two were similar mesenchymal neoplasms occurring at different loci with in the bronchial tree ${ }^{(5)}$ Bronchial derived hamartomas, in most reported cases have shown a very slow growth rate over a period of years and remained clinically silent. The cause of bronchial obstruction is not always malignant. The location of the tumor and severe obstructive changes necessitated a pneumonectomy which resulted in a cure of the patient.The prognosis in completely resected lesion is excellent .

\section{Conclusion:}

Pulmonary hamartoma,though a benign tumor,can rarely grow rapidily and cause severe obstructive changes.Our case report illustrate the cause of bronchial obstruction is not always malignant.The prognosis in compleletely resected lesion is excellent

\section{References}

[1] J.Murray,D.Kielkowski,and G.Leiman,"The Prevalence and age distribution of peripheral pulmonary hamartomas in adult males.An autopsy_based study”.South African medical journal,Vol 79,no-5,pp.247-249,1991.

[2] B.G.Cosio,V.Villena,J.Echava-sustaeta et al;"'Endobronchial hamartoma",chest,vol 122,no1,pp.n202-205,2002

[3] Van den bosch JM,Wagenaar SS,Corrin B,et al.Mesenchymoma of the lung(So called hamartoma):A review of 154 parenchymal and endobronchail cases. Thorax 1987;42:790-3.

[4] Ramirez-Chavez G,celard p,Gamondes JP,et al.pulmonary hamartoma:Apropos of 49 surgically treated cases. Sem Hop paris 1984;60:1181-4.

[5] Tomashefski JF.Benign endobronchial mesenchymal tumors.Their relationship to parenchymal pulmonary hamartomas.Am J surg pathol 1982;6:531-40. 